\title{
La organización institucional de la Comunidad Autónoma de Andalucía*
}

\author{
José María Morales Arroyo \\ Catedrático de Derecho Constitucional \\ Universidad de Sevilla
}

\section{INTRODUCGIÓN}

El proceso de reforma estatutaria en sus diferentes etapas ha dejado al descubierto campos temáticos cuya delimitación ha suscitado un intenso debate y arduos enfrentamientos entre las distintas fuerzas políticas. En cambio, otros temas, como el de la reforma de las instituciones de autogobierno, no se han llegado a colocar entre las cuestiones disputadas. Un silencio que tiene poco sentido si se considera que la reforma de los Estatutos de Autonomía persigue un avance y un fortalecimiento del autogobierno, y esto sólo se conseguirá con una mejora del funcionamiento de las instituciones de cada Comunidad Autónoma.

A este respecto, Andalucía no ha sido la excepción. Salvo alguna contraposición sobre la conveniencia o no de limitar estatutariamente los mandatos del Presidente de la Junta de Andalucía o sobre la necesidad de obligar a que se convoquen las elecciones autonómicas separadas de otras consultas electorales, el consenso ha reinado en torno al alcance de las reformas de las disposiciones que definen el modelo institucional.

No obstante existen algunas premisas que condicionan en este campo el debate de las reformas autonómicas y que conviene recordar

La evolución del Estado autonómico ha configurado un modelo bastante homogéneo en la organización institucional, en el que coinciden sus princi-

\footnotetext{
* Texto de Ponencia presentado para Instituto García Oviedo, JORNADAS DE ESTUDIO SOBRE LA REFORMA DEL ESTATUTO DE AUTONOMÍA PARA ANDALUCÍA. Universidad de Sevilla, enero 2007; se ha mantenido en lo básico el contenido de la intervención.
} 
pales rasgos: Existe una Cámara representativa, electa por el cuerpo electoral autonómico respetando criterios de representación territorial, con capacidad legislativa y presupuestaria y con la función de legitimar originariamente al Consejo de Gobierno, que, posteriormente, controla. El Consejo de Gobierno ejerce facultades ejecutivas y administrativas bajo la dirección de un Presidente, que es investido por la Asamblea legislativa. La diferencia más radical entre los modelos institucionales de las Comunidades Autónoma ha quedado reducida al reconocimiento de la facultad presidencial para disolver discrecionalmente la institución parlamentaria y convocar procesos electorales; una distinción que en la práctica puede (y quizás debe) desaparecer si se impone la tendencia marcada por la reforma del Estatuto de Autonomía de la Comunidad Valenciana.

El modelo común se imponía como un imperativo constitucional en el proceso de reforma del Estatuto de Autonomía de Andalucía, en la medida que el legislador no podía despreciar las guías fijadas por el artículo 152 de la Constitución. Pero, además, la práctica institucional definida a partir de la regulación estatutaria durante más de veinte años se puede decir que ha moldeado un modelo institucional con sus propias inercias, que no ha revelado grandes disfunciones. El régimen parlamentario andaluz, en el marco del artículo constitucional, se configuró recurriendo al reconocimiento de una doble responsabilidad política del Presidente de la Junta y del Consejo de Gobierno ante el Parlamento de Andalucía. Las normas estatutarias originarias diseñaron un modelo con una cierta tendencia a reforzar la posición del Parlamento en el entramado institucional, utilizando, para ello el mecanismo de concederle el ejercicio de competencias medulares de la Comunidad Autónoma. Además, se confería a la voluntad parlamentaria la potestad de ser el origen y marcar el final del mandato del Consejo de Gobierno, al tiempo que, por el contrario, se privaba al Presidente de la Junta de la capacidad discrecional para disolver la Cámara ${ }^{1}$. La realidad marchó por derroteros bien distintos y, como en los sistemas políticos de nuestro entorno, el reforzamiento institucional del Ejecutivo ha venido provocando un paralelo debilitamiento y transformación del Parlamento.

Por último, los rendimientos y la estabilidad conferida al sistema andaluz por el funcionamiento de las instituciones han resultado más positivos que ne-

\footnotetext{
${ }^{1}$ Según se especificaba en los artículos 30, 35.3, 37 y 39 del Estatuto de 1982 y las prácticas que, a partir de sus preceptos, se configuraron.
} 
gativos, destacando en el debe sólo circunstancias como la falta de alternancia política, la tendencia a la confusión de los procesos electorales y el bloqueo que se verificó coyunturalmente durante la Cuarta Legislatura.

Tomadas en su conjunto, estas premisas explican el corto alcance de la reforma en los preceptos estatutarios dedicados a las instituciones de autogobierno en el marco de un texto normativo que casi ha multiplicado por tres sus preceptos. Los Títulos II y III del Estatuto de 1982 sumaban 22 y 8 artículos, respectivamente, mientras que los novedosos Títulos IV y V abarcan desde el artículo 99 al 155. El gran salto se produce en la regulación de los temas relacionados con la justicia que doblan su articulado (de 8 a 16) a la vez que aumentan su complejidad; mientras que el crecimiento en las disposiciones sobre las tradicionales instituciones de autogobierno se debe especialmente al incremento de los preceptos dedicados a la producción de normas (Capítulo II, del Título IV, con 9 artículos frente a los 3 artículos con los que contaba el Capítulo $2^{\circ}$, del Título II) y a la introducción cinco artículos; los artículos que en el nuevo Capítulo VI se dedican a las "Otras instituciones de autogobierno”. Estos juegos numéricos en sí mismos no tienen un singular significado porque no se trata de una de las partes que, como se apuntaba, más se desarrollan en el corpus del Estatuto y porque se ha impuesto una opinión generalizada a que cuestiones como la disolución parlamentaria o los órganos auxiliares de autogobierno se recogiesen en el texto del Estatuto tanto por respeto a la naturaleza de la norma que debía acoger su regulación, como por razones de dignidad institucional para las instituciones que reciben reconocimiento estatutario. Pero, de partida, supone un dato interesante si se considera que introduce un importante grado de rigidez en el futuro funcionamiento de las instituciones. Es decir, la positivación resalta la posición de las instituciones reguladas y coloca su existencia, parte de su estructura y de sus reglas de funcionamiento fuera del marco de la lucha política confiriéndoles estabilidad, a partir de aquí, los posibles defectos que se detecten en su funcionamiento, cuando se deriven del diseño estatutario no se van a poder corregir más que siguiendo los "sencillos" trámites dispuestos para la reforma estatutaria.

\section{LOS ASPECTOS MÁS SIGNIFICATIVOS EN LA REFORMA DE LAS INSTITUCIONES}

El germen de las propuestas de reforma en las cuestiones institucionales se localizaban ya en los Acuerdos de la Ponencia de la Comisión de Desarrollo Estatutario y, según se ha dicho, tenían como referencia de partida la op- 
ción diseñada por el legislador estatuyente en el año 1982, en el amplio espacio que dejaba el artículo 152 de la Constitución.

Como una primera misión, la reforma ha asumido la tarea de corregir las condiciones en las que se regulaban ciertas instituciones en el articulado originario, que, como la práctica había demostrado, daban lugar a consecuencias disfuncionales e inaceptables para el funcionamiento de una Comunidad Autónoma como la de Andalucía. En este sentido, no se ha discutido la necesidad de ciertas reformas.

Primero, de manera acertada se ha optado por permitir la ampliación de la base democrática del Parlamento de Andalucía con la simple ampliación del número de miembros integrantes de la Cámara más allá de los 109 diputados (art. $101 \mathrm{EAA}$ ). El instrumento para ello debe ser la Ley Electoral de Andalucía. Con esta medida, superando problemas técnicos de distribución de diputados en un ajustado Salón de Plenos, se puede conseguir un aumento de la ratio representativa entre la población andaluza y los miembros de la Cámara territorial. Para que se vean con claridad los problemas y los resultados futuros de la reforma estatutaria basta comparar los datos de los Parlamentos de las Comunidades Autónomas más pobladas. El Parlamento de Cataluña se integra por 135 diputados que representan a una población de 7.134.697 habitantes y son elegidos por un cuerpo electoral de 5.321 .274 electores (año 2006), la Asamblea de Madrid se compone de 120 diputados que representan a 6.008.908 habitantes y son elegidos por 4.317.171 electores (año 2007) y el Parlamento de Andalucía, conforme a las antiguas previsiones estatutarias, se integra por 109 diputados, que representan a una población de 7.975.672 habitantes y son elegidos por 6.052.012 electores (año 2004). Pues bien, sin detenerse en los problemas de la mejora de la proporcionalidad ni de la distorsión que supone el reparto entre circunscripciones de uno u otro tipo, cada diputado en Cataluña representa a una cuota ideal de 52.849 habitantes y de 39.417 electores y cada parlamentario de Madrid asume la representación de 50.074 habitantes y de 35.976 integrantes del cuerpo electoral autonómico, mientras que cada diputado en Andalucía representa a una fracción de 73.171 habitantes y 55.523 electores. La actual realidad distorsiona bastante el pronunciamiento del artículo 100 del EAA, ya que difícilmente se puede aceptar que en su composición actúe como una representación fidedigna del pueblo andaluz.

Segundo, los artículos 104 y 105 del EAA han liberado a los legisladores autonómicos de la vinculación automática del sistema electoral previsto para elegir a los diputados andaluces y el sistema del Congreso de los Diputados, que se derivaba de la aplicación del antiguo artículo estatutario (28.2). 
Tercero, se han establecido las bases para permitir con el respaldo estatutario que el Presidente de la Junta pueda proceder a la disolución discrecional y anticipada del Parlamento, sacando esa facultad del marco legal al que se circunscribía. El Estatuto, de un lado, define con mayor detalle la extensión y finalización del mandato de los diputados andaluces, incluyendo la disolución de la Cámara entre las causas de la extinción del mismo (art. 101.2), y, de otro, dedica un detallado artículo ?el 127? a la forma y límites con los que el Presidente de la Comunidad Autónoma puede proceder a la disolución del Parlamento.

En conexión con la anterior medida, se ha suprimido la fórmula con la que se solventaban los bloqueos en el proceso de elección del titular de la Presidencia de la Junta. La solución se conecta con los mecanismos habituales de disolución parlamentaria, suprimiendo la poco democrática y dudosamente constitucional cláusula residual que investía automáticamente al candidato del partido que tuviese mayor número de escaños, aunque no gozase de la confianza de la Cámara o incluso con el voto en contra de la mayoría del Parlamento de Andalucía (antiguo art. 37.2). El artículo 118, en el párrafo 3o, in fine dispone una vía de disolución automática del Parlamento, con convocatoria de elecciones del Presidente de la Junta en funciones, si no se hubiese investido un candidato después de transcurridos dos meses desde la primera e infructuosa votación; con ello se adopta el modelo seguido por el artículo 99.5 de la Constitución y recogido en el resto de los Estatutos de Autonomía, con la sola excepción del Estatuto de Autonomía para Castilla-La Mancha.

A estas modificaciones, en cuya inserción ha regido el consenso y que, sin duda, van a mejorar la dinámica del modelo parlamentario, pocas novedades se pueden añadir. Formalmente, se aprecia una mejora en la sistematización del articulado y sus contenidos que se puede considerar ejemplo de una buena técnica legislativa.

En este sentido, se puede mencionar el esfuerzo realizado para completar y ordenar las normas referentes a la organización y formación del Parlamento de Andalucía, sus competencias y prerrogativas. Entre las novedades que pretenden un cierto reforzamiento del papel institucional de la Cámara se pueden señalar la garantía de unos períodos mínimos de sesiones ordinarias para que ocupen al menos ocho meses (art. 103.3 EAA), una definición más acabada de las manifestaciones de la autonomía parlamentaria (art. 102 EAA) y una completa enumeración de las funciones de la Asamblea, con la introducción de novedades como la de "orientación e impulso de la acción del Consejo de Gobierno” (art. 106 EAA). 
Un esfuerzo similar se detecta en la configuración normativa de la Presidencia de la Junta y del Consejo de Gobierno, que se ha realizado en los Capítulos III y IV del Título IV de la reforma estatutaria. La reforma con la separación de la normativa sobre el Ejecutivo andaluz en dos capítulos separados está reconociendo el papel institucional propio que ha adquirido en la vida política andaluza y en la estructura de los órganos de autogobierno el Presidente de la Junta de Andalucía.

Por lo demás, entre las novedades, además de la señalada con respecto al proceso de investidura presidencial, se destacan la creación un Capítulo V, a semejanza del Título V de la Constitución, que se dedica a "las relaciones entre el Parlamento y el Consejo de Gobierno" y que incluye la responsabilidad política del Consejo de Gobierno (art. 124 EAA) y regula agrupadamente la cuestión de confianza (art. 125 EAA), la moción de censura (art. 126 EAA) y la disolución del Parlamento de Andalucía (art. 127 EAA).

La breve panorámica realizada sobre los tradicionales órganos de autogobierno debe cerrarse con una exposición de las cuestiones que a nuestro juicio dentro de la reforma estatutaria se espera que vayan a tener una mayor repercusión en la futura dinámica de las instituciones andaluzas.

El panorama de las instituciones se verá casi con seguridad alterado por la tendencia a la parificación sobre criterios de género en la composición de los órganos de autogobierno de la Comunidad Autónoma. Esta opción estatutaria tiene un mayor alcance, no se limita a la composición de los órganos de la Comunidad. A modo de ejemplo, en el artículo 10, párrafo $2^{\circ}$, cuando se fijan los objetivos básicos de la Comunidad Autónoma se incluye como un mandato a los poderes públicos el de propiciar "la efectiva igualdad del hombre y de la mujer andaluces, promoviendo la democracia paritaria y la plena incorporación de aquélla en la vida social, superando cualquier discriminación laboral, cultural, económica, política y social”.

Con respecto a la formación y el funcionamiento de las instituciones de autogobierno, tras la reforma estatutaria, el articulado ofrece cobertura estatutaria a la norma legal sobre paridad electoral (art. 105.2), establece como criterio vinculante para el Parlamento de Andalucía la búsqueda del equilibrio entre hombres y mujeres en la formación de los órganos de nombramiento parlamentario (art. 107) y dispone la realización de estudios de impacto de género sobre el contenido de las leyes y de los reglamentos aprobados por la Comunidad Autónoma (art. 114). 
Sobre el nombramiento de órganos, resulta curioso que no se haga una mención expresa a la búsqueda de la paridad o del equilibrio cuando se trata de la composición del Consejo de Gobierno, lo que es más llamativo cuando precisamente dicho criterio se ha utilizado para la formación del Consejo en la Séptima Legislatura tras las elecciones de marzo de 2004. El silencio del articulado del proyecto estatutario se puede interpretar en el sentido de que rige la plena libertad de configuración del Presidente de la Junta en la designación de los Consejeros (art. 117.1 EAA), quedando a la valoración y voluntad de cada Presidente la formación o no de un gobierno paritario o equilibrado. O, en el mejor de los casos, que la limitación se introduzca puntualmente en la Ley sobre el régimen jurídico y administrativo del Consejo de Gobierno, como hace actualmente el artículo 18.3 de la Ley 6/2006, de 24 de octubre; con esta solución el criterio de la paridad pierde capacidad de resistencia como principio organizativo, al carecer de la garantía derivada de la rigidez estatutaria.

Por otro lado, el criterio de nombramiento equilibrado y paritario de los órganos se va a encontrar enfrente y deberá superar el hábito de la fijación de cuotas por grupos políticos en la formación de los órganos colectivos. Lo que, no resulta fácil, si se tiene presente las dificultades que han jalonado el reciente proceso de nombramiento de los cargos de adjunto al Defensor del Pueblo de Andalucía. No obstante, estos titubeos se pueden considerar contratiempos iniciales en la aplicación de una norma que la dinámica política deberá imponer con naturalidad en la práctica democrática y parlamentaria; la propia previsión estatutaria coloca el deber por encima de la voluntad de las fuerzas políticas y, al no poder eludirlo, cada partido en cada ocasión se verá abocado ante la opinión pública a justificar las razones de su resistencia al cumplimiento.

Por último, sobre el criterio para la formación de las listas electorales continúa abierto el debate de su compatibilidad con el ejercicio de los derechos fundamentales del artículo 23 y del artículo 22, en su conexión con el artículo 6, todos de la Constitución; aunque se pueden salvar más razonablemente las dudas de constitucionalidad, en la medida que se verifica el expreso reconocimiento estatutario en el marco de políticas dirigidas a promover "condiciones para que la libertad y la igualdad del individuo y de los grupos en que se integran sean reales y efectivas", a remover "los obstáculos que impidan o dificulten su plenitud" y a fomentar "la calidad de la democracia facilitando la participación de todos los andaluces en la vida política, económica, cultural y social" (art. 10.1 EAA, en relación con el art. 9.2 de la CE). 
La progresiva puesta en práctica de esas reglas tendrá como resultado futuro un importante cambio en la vida política e institucional de nuestra Comunidad y originará una sustancial e interesante transformación en el perfil, en la fisonomía, de los órganos de autogobierno andaluces.

También se puede considerar una innovación el evidente refuerzo de la tendencia al predominio del ejecutivo. La racionalización entendida como juridificación de las relaciones entre los órganos superiores de la Comunidad Autónoma se ha considerado una solución que beneficia a los gobiernos frente a las instituciones parlamentarias. En la práctica, en el marco de las instituciones de autogobierno de las Comunidades Autónomas se ha constatado que los instrumentos dispuestos para la exigencia de responsabilidad política y, en su caso, para poner fin a los bloqueos en el sistema político se vienen mostrando ineficaces. Sólo parcialmente el recurso a la disolución anticipada del Parlamento ha demostrado alguna viabilidad para resolver conflictos graves. El ejemplo más evidente de esa afirmación en la reciente historia política de Andalucía se encuentra en el bloqueo que representó la Cuarta legislatura, con un gobierno en minoría y una oposición con mayoría parlamentaria que, por cuestiones estratégicas, optó por bloquear la labor del ejecutivo sin sustituirlo; la disolución anticipada y la llamada al electorado en 1996 marcó la vía para la solución del conflicto.

En honor a la verdad, la reforma deja entrever un esfuerzo en la línea de reforzar la posición del Parlamento de Andalucía. Como se ha apuntado, se refuerzan los rasgos de su autonomía (art. $102 \mathrm{EAA}$ ), se reservan a la autoformación los elementos de su organización y funcionamiento (art. 103 EAA), se libera el régimen electoral de ataduras inadmisibles desde la perspectiva de un ente titular de autonomía política (art. $104 \mathrm{EAA}$ ), se deja abierta la puerta para aumentar su representatividad con el aumento de sus miembros (art. 101 EAA) y se dibujan sus competencias de una manera minuciosa, añadiendo alguna como la de orientación e impulso de la acción de Gobierno (art. 106 EAA), que hasta ahora se recogían en disposiciones legales o se configuraban a través de una práctica de casi veinticinco años. Pero junto a todo esto, el reconocimiento del ejercicio de la competencia de "dirección política de la Comunidad Autónoma” al Consejo de Gobierno (art. 119.2 EAA), además del aumento de sus potestades legislativas gubernamentales y de la atribución a la Presidencia de la capacidad para disolver anticipadamente la Cámara legisladora, marcan una tendencia opuesta al reforzamiento institucional del legislativo. El modelo institucional consolidado tras la práctica de 25 años confirma esta afirmación. 
A este respecto se ha introducido en el Capítulo II del Título IV, dedicado a la "Elaboración de las normas", la facultad del Consejo de Gobierno para dictar normas con rango de ley, bien previa delegación del Parlamento de Andalucía (art. 109 EAA), bien en casos de extraordinaria y urgente necesidad (art. 110 EAA). Esa posibilidad supone un refuerzo de la posición del Ejecutivo andaluz en el proceso de creación del Derecho en nuestra Comunidad y, a la inversa, la inclusión de este tipo de normas supone una perdida de capacidad normativa para el Parlamento, en la medida que la ley convive y compite con las normas de producción gubernamental.

No obstante, la regulación estatutaria del Decreto legislativo se puede considerar un elogiable avance que permitiría el recurso garantizado a un instrumento de colaboración legislativa entre Parlamento y Consejo de Gobierno. En el caso de este tipo normativo, resulta conocido que la potestad delegada sigue siendo titularidad del órgano cedente, el Parlamento de Andalucía, que es el que fija los términos de su ejercicio posterior por el Ejecutivo, el que controla la regularidad del uso del mandato por el delegado y el que, respetando ciertas condiciones, puede recuperar la capacidad legisladora sobre la materia transferida o sencillamente derogarla con una ley posterior. La inseguridad del ejercicio de la delegación sin pautas normativas claras se corrige con la previsión de la mecánica y los límites dentro del articulado estatutario, con lo que se evitan fraudes que pongan en peligro las reservas legislativas fijadas en el Estatuto y eventuales abusos gubernamentales. Es más, el recurso a esa vía de colaboración normativa se va a terminar convirtiendo en una necesidad si continúa la práctica de acompañar anualmente la Ley presupuestaria con una "Ley de acompañamiento" que trastoca parte del ordenamiento preexistente; a consecuencia de esa práctica se produce una acumulación y superposición de reformas en leyes andaluzas fundamentales sobre hacienda, régimen administrativo, funcionarios, reglas sociales, etc., que enmaraña el entendimiento sistémico del ordenamiento de nuestra Comunidad Autónoma. Una delegación a tiempo para refundir textos legislativos puede ser una magnífica salida para depurar y simplificar ciertas ramas normativas andaluzas ${ }^{2}$.

\footnotetext{
${ }^{2}$ A este respecto quizás, no perdiendo de vista lo que ha sido la práctica de la delegación legislativa tanto en el ámbito estatal, como en las Comunidades Autónomas que lo han usado, tiene pleno sentido prever este procedimiento normador para la refundición de textos, pero no tanto para la creación de textos nuevos o articulados a partir de leyes de bases de procedencia parlamentaria. Ciertamente hasta el momento no se ha hecho gran uso de esta modalidad regulada en la Constitución, mientras que ha sido frecuente el recurso a la delegación para la creación de textos refundidos, tanto estatales como autonómicos. En el marco estatal, el auge de la delega-
} 
Mientras que la aceptación del decreto-ley en el ordenamiento autonómico suscita una mayor inquietud. La justificación de este tipo de normas se ha asentado en un doble criterio; de un lado, el cumplimiento de los elementos del supuesto de hecho habilitante y de la imposibilidad de dar respuesta normativa en unos plazos razonables a través de la producción de la Ley; y de otro lado, la concesión al gobierno de un instrumento normativo fuerte y eficaz para el cumplimiento de ciertas políticas públicas que reclama la dinámica del Estado social y democrático contemporáneo.

La capacidad normativa del Consejo de Gobierno sólo se puede valorar insertada en la dinámica de la producción legislativa seguida por la Comunidad Autónoma de Andalucía. El proceso de atribución competencial con el que se conformaron las Comunidades Autónomas ha hecho que el ejercicio de la potestad legislativa haya sido constante, pero reducido en sus frutos. En este aspecto la historia de la Comunidad Autónoma de Andalucía no ha sido una excepción; el Parlamento ha aprobado 220 leyes hasta el año 2006, lo que supone, sin detenerse en las particularidades que justifican la existencia de años más productivos y menos productivos, una producción media que oscila entre 8 y 9 leyes por año. Si en el futuro el número de los decretos-leyes aprobados en el marco autonómico siguiesen los porcentajes que rigen en la política estatal, estaríamos hablando de un número que oscilaría entre el 20 y el 30 por ciento de las normas con rango de ley aprobadas en nuestra Comunidad. Se quiera o no supondría un golpe para el ejercicio de una de las principales funciones de todo Parlamento, incluyendo los autonómicos, y una disminución del control de la opinión pública sobre los contenidos de unas normas que se aprueban a través de un procedimiento más opaco y en el que el debate y la supervisión parlamentaria se verifica a posteriori.

En oposición a este argumento se puede mantener que la razón que justifica la previsión y la posterior producción de los decretos-leyes no sería tanto el número de los dictados, sino la necesidad y la urgencia que impelen a su publicación. Pero, a este respecto también se puede apuntar que los argumentos que justifican el reconocimiento de la potestad de crear decretos-leyes en el ordenamiento estatal no son sin más trasladables al ámbito jurídico au-

ción mediante bases se produce en las Segunda y Tercera Legislaturas de las Cortes Generales, períodos en los que se aprueban 15 decretos legislativos a través de los que se trasponen reglas recogidas en normas europeas, como consecuencia de la entrada de España en las Comunidades Europeas; mientras que en las Legislaturas Primera y Quinta se aprobó un solo decreto legislativo a partir de normas de bases y en la Cuarta cuatro. 
tonómico. En este sentido, el factor tiempo en la producción de una norma con rango de ley debe ponderarse en el marco de una Cámara y un procedimiento legislativo menos complejo que el dispuesto para las Cortes Generales. Es decir, la lentitud del procedimiento legislativo se presenta como una coartada razonable cuando nos referimos al que transita por unas Cortes bicamerales en las que se suceden diferentes "lecturas" de la iniciativa legislativa tanto en el Congreso de los Diputados como en el Senado; en cambio, es más que discutible ese argumento, cuando se está haciendo referencia a Asambleas como las autonómicas, menos numerosas, con sistemas de partido simplificados, más cercanos y abiertos a los ciudadanos representados y con unos procedimientos de urgencia legislativa que permiten una tramitación y aprobación de una ley en plazos de tiempo aceptables.

En conclusión, la justificación de la premura temporal sería difícilmente demostrable en el ámbito autonómico, resultando, además, previsible que la entrada del Consejo de Gobierno en la producción de normas con rango de ley venga a suponer una disminución de las funciones legisladoras del Parlamento y de las de control político del ejecutivo.

A ello debe añadirse que tras la aprobación y entrada en vigor del Estatuto nos encontraríamos en una situación de "cierta alegalidad" desde una perspectiva procedimental. La vigente Ley del Gobierno de Andalucía, Ley 6/2006, de 24 de octubre, cuya elaboración ha coincidido en el tiempo con el debate de los contenidos estatutarios, ha omitido cualquier referencia a la aprobación de normas con rango de Ley por el Consejo de Gobierno; ni en el artículo 27 de la Ley, dedicado a las competencias del Consejo, ni en el artículo 46, cuando se clasifican las formas que deben adoptar las decisiones del mismo, se mencionan a los decretos-leyes ni a los decretos legislativos. Mientras que el Capítulo I del Título VI se dedica al modo en el que se debe ejercer la iniciativa legislativa y al cómo aprobar las disposiciones reglamentarias ${ }^{3}$. El problema de la cobertura competencial se podría suplir con la atribución directa hecha por las normas estatutarias en combinación con la disposición residual del apartado 20 del mencionado artículo 27; pero el silencio en el precepto dedicado a las formas de manifestación de las decisiones del ejecutivo andaluz sólo se solventaría con una reforma del precepto que diera cabida a las fuentes creadas por vo-

\footnotetext{
${ }^{3}$ Las normas paralelas de la Ley 50/1997, de 27 de noviembre, reguladoras del Gobierno, disciplinan mínimamente la creación de los decretos leyes y legislativos, como competencia del Consejo de Ministros (art. 5.1,c) y como una de las formas jurídicas que adoptan las decisiones del Gobierno (art. 25.2,a)
} 
luntad estatutaria. Incluso, resultaría recomendable una mención expresa al proceso de elaboración de estas peculiares disposiciones gubernamentales. No se puede afirmar que estas carencias impidan, llegado el caso, el ejercicio de las competencias estatutarias al Consejo de Gobierno; pero, unas mínimas exigencias de seguridad jurídica llevan a solicitar un mayor rigor y detalle en la regulación de la cuestión dentro de las normas de desarrollo estatutario.

\section{LAS INSTITUCIONES QUE HAN RECIBIDO RECONOCIMIENTO ESTA- TUTARIO}

En su evolución las Comunidades Autónomas han superado el limitado esquema institucional que fijaba la Constitución en su artículo 152 y que se generalizó como consecuencia de la aplicación de los Acuerdos Autonómicos de 1981. La práctica dejó al descubierto que resultaba necesaria la creación y el concurso de una serie de nuevos órganos para que la vida autonómica se desarrollara con fluidez y un cierto equilibrio institucional.

La principal objeción a este modo de proceder provenía de que tales órganos se creaban en un margen de la legalidad, fuera del articulado estatutario y en ocasiones contra lo que era el tenor literal de algún precepto de la norma institucional básica o de la propia Constitución; como ocurría, por ejemplo, con el mandato establecido en el artículo 153.1,d de la Constitución a favor de que el control económico y financiero de las actividades de las Comunidades Autónomas correspondiese al Tribunal de Cuentas. Esa incómoda situación contradecía la importancia que poco a poco habían ido adquiriendo esas instituciones y la dignidad que merecían por su labor en el entramado orgánico de cada Comunidad Autónoma.

En el caso andaluz, incluso, el artículo 44 del Estatuto inicial atribuía al Consejo de Estado las competencias de control sobre la actuación de la Administración andaluza, mientras que el artículo 70 de la misma norma reiteraba que el control financiero de los poderes públicos de Andalucía pertenecía al Tribunal de Cuentas. La necesidad y la funcionalidad del modelo autoorganizativo de la Junta de Andalucía obligaron a que a través de la Ley del Parlamento de Andalucía 1/1988, de 17 de marzo (reformada por Ley 2/1996), se crease la Cámara de Cuentas y que mediante la Ley 8/1993, de 19 de octubre (sustituida por Ley del Parlamento de Andalucía 4/2005), se constituyese y comenzase a funcionar el Consejo Consultivo. 
A estos órganos, excluidos del articulado estatutario, se han añadido el Consejo Económico y Social de Andalucía, creado por Ley 5/1997, y, más recientemente el Consejo Audiovisual (creado por Ley andaluza 1/2004, de 17 de diciembre).

La dignidad de los órganos mencionados requería que, una vez que se ha iniciado una profunda reforma estatutaria, se aprovechase la ocasión para incluir referencias a esas instituciones en su articulado, confiriendo a su existencia una garantía normativa especial. En el Capítulo VI del Título IV, bajo la rúbrica de "Otras instituciones de autogobierno", se han regulado las instituciones de control público que hasta ahora han quedado fuera del Estatuto de Autonomía.

El Consejo Consultivo en el artículo 129, se ha definido como órgano consultivo del Consejo de Gobierno y de la Administración andaluza, órgano de asesoramiento de las corporaciones locales y su administración y de los órganos sometido a Derecho público. En el precepto se incluye tanto una reserva legal que alcanza a su composición, competencia y funcionamiento, como una garantía estatutaria de autonomía orgánica y funcional. Con similar estructura, el artículo 130 se dedica a la Cámara de Cuentas, considerada el órgano de control externo de la actividad económica y presupuestaria de la Junta de Andalucía, los entes locales y del sector público andaluz; aunque, en este caso, el reconocimiento de una reserva legal para su definición, se acompaña con un expreso pronunciamiento sobre su dependencia orgánica del Parlamento de Andalucía.

Al reconocimiento estatutario del Consejo Económico y Social de Andalucía se destina el actual artículo 132 y al Consejo Audiovisual el 131.

Con el reconocimiento en la norma institucional básica de Andalucía se consigue el cierre de una especie de círculo virtuoso, configurado con una dinámica inversa. En vez de partir de un reconocimiento estatutario que garantizase su existencia y rasgos básicos frente a una posterior intervención del legislador, se ha producido una previa definición legal de estas instituciones de autogobierno, que con posterioridad ha marcado su reconocimiento y configuración estatutaria.

\section{UNA REFERENCIA A LAS COMPETENCIAS SOBRE JUSTICIA EN EL NUEVO ESTATUTO}

La confusa denominación que recibe el Título V del Estatuto, con evidente inspiración en la norma homónima recientemente aprobada para Ca- 
taluña, no nos debe llevar a engaño. En realidad, no se puede hablar de "un poder judicial de Andalucía" en la medida que se ha terminado imponiendo la interpretación constitucional de que la jurisdicción es única y se ejerce sobre todo el territorio del Estado. En consecuencia, la Junta de Andalucía no interviene directamente en el ejercicio de la función jurisdiccional y sólo se ha hecho cargo del ejercicio de ciertas facultades relacionados con parte del personal de la Administración de justicia y con los medios materiales por ésta utilizados en el marco del territorio de la Comunidad Autónoma.

En este sentido, pese a la confusa redacción del artículo 152 del texto constitucional, resulta evidente que el Tribunal Superior de Justicia de Andalucía no es órgano de la Junta de Andalucía. Esta separación estructural se ha remarcado en la nueva organización del articulado tras la reforma estatutaria; las referencias al Tribunal Superior de Justicia salen del Título IV, dedicado a la organización institucional de la Comunidad Autónoma, para recogerse en el Título V, sobre "el Poder Judicial en Andalucía" (al TSJA se refieren los arts. 140, 142 y 143). Pero, además, funcionalmente, el nuevo Estatuto pretende atribuir a la Comunidad Autónoma directamente competencias, cuyo desarrollo y delimitación efectiva queda a la soberanía de la Ley Orgánica del Poder Judicial, que continúa actuando así en la mayoría de las ocasiones como, en términos de Jiménez Asensio, "ley competencial"4.

Una reflexión parecida cabe hacer sobre el Consejo de Justicia de Andalucía, al que se dedica un Capítulo completo, el Segundo, con un extenso artículo 144. Este novedoso órgano ha pasado durante la tramitación parlamentaria de ser definido como "el órgano de gobierno del Poder Judicial de Andalucía”, que deberá funcionar en los términos que en su día dispongan los preceptos de la Ley Orgánica del Poder Judicial como "órgano desconcentrado del Consejo General" de ese poder, a ser catalogado de manera incierta como el "órgano de gobierno de la Administración de Justicia en Andalucía". El órgano se compondrá por el Presidente de Tribunal Superior de Justicia de Andalucía, que lo presidirá, y por un número indeterminado de vocales, es-

${ }^{4}$ La noción aparece en Rafael Jiménez Asensio: "Reforma de la Administración de justicia y Comunidades Autónomas", en Parlamento y Constitución, 7 (2003), p. 13.

${ }^{5}$ Para la primera conceptuación art. 142.1 de la proposición aprobada por el Parlamento de Andalucía y tomada en consideración por el Congreso de los Diputados; para la definición que permanece tras la negociación, art. 144.1 del texto aprobado por el 2 de noviembre por el Pleno del Congreso. 
cogidos entre jueces, magistrados, fiscales y juristas de reconocido prestigio; en la elección de los vocales se establece como criterio la participación del Parlamento de Andalucía y se establece una remisión a la Ley Orgánica para la fijación del definitivo sistema de su selección.

Las funciones del nuevo órgano se fijan en la Ley Orgánica del Poder Judicial, en el Estatuto de Autonomía y en las leyes del Parlamento de Andalucía, asumiendo, además, aquellas otras que les delegue el Consejo General del Poder Judicial. En todo caso, en el apartado $4^{\circ}$ del artículo 144 aparece un listado de competencias mínimas, cuyo ejercicio se coordina con otras desarrolladas por el Consejo General del Poder Judicial y se definen en las normas estatales sobre Poder Judicial.

El Consejo de Justicia de Andalucía se puede considerar órgano de la Comunidad Autónoma en el mismo sentido que el Consejo General del Poder Judicial se puede considerar órgano estatal. Se trata de un órgano reconocido y garantizado estatutariamente, en su formación deberá participar el Parlamento de Andalucía, parte de sus funciones se atribuyen mediante mandato estatutario, además de a través de decisiones concretas del legislativo andaluz y, por último, su campo de actuación se refiere a la Administración de Justicia de Andalucía, que hasta el momento, y desde la atribución competencias realizada por el Real Decreto 142/1997, de 31 de enero, constituye una de de las parcelas sobre las que la Junta de Andalucía ejerce sus competencias en el ámbito de la justicia. Lo que resulta más difícil es su catalogación como institución de autogobierno, en la medida que no parece destinado a formar la voluntad de la Comunidad Autónoma; en todo caso, en un sentido muy laxo cabría apuntar que participa e interfiere sobre algunas parcelas de las competencias que sobre administración y medios materiales de justicia desarrolla la Junta de Andalucía.

Por último, no cabe descartar que en el futuro el Consejo entre en competencia o colisione con las instituciones de la Junta de Andalucía, cuando ejercite algunas competencias atribuidas por el Estatuto o asumidas a partir de concesiones legales. Así, por ejemplo, la competencia que el artículo 52.2 del antiguo texto estatuario atribuía a la Comunidad Autónoma de "fijar la delimitación de las demarcaciones territoriales de los órganos jurisdiccionales de Andalucía, de conformidad con la Ley Orgánica del Poder Judicial", se incorpora al nuevo Estatuto en el art. 151; pero, al mismo tiempo, también se ha dispuesto la participación del Consejo de Justicia de Andalucía con un informe (arts. 144.4,g y 151.1). 